\title{
Towards a high-energy theory for the Higgs phase of gravity
}

\author{
Michael L. Graesser, ${ }^{1}$ Ian Low, ${ }^{2}$ and Mark B. Wise ${ }^{1}$ \\ ${ }^{1}$ California Institute of Technology, 452-48, Pasadena, California 91125, USA \\ ${ }^{2}$ School of Natural Sciences, Institute for Advanced Study, Princeton, New Jersey 08540, USA
}

(Received 20 October 2005; published 28 December 2005)

\begin{abstract}
Spontaneous Lorentz violation due to a time-dependent expectation value for a massless scalar has been suggested as a method for dynamically generating dark energy. A natural candidate for the scalar is a Goldstone boson arising from the spontaneous breaking of a $U(1)$ symmetry. We investigate the lowenergy effective action for such a Goldstone boson in a general class of models involving only scalars, proving that if the scalars have standard kinetic terms then at the classical level the effective action does not have the required features for spontaneous Lorentz violation to occur asymptotically $(t \rightarrow \infty)$ in an expanding Friedman-Robertson-Walker universe. Then we study the large $N$ limit of a renormalizable field theory with a complex scalar coupled to massive fermions. In this model an effective action for the Goldstone boson with the properties required for spontaneous Lorentz violation can be generated. Although the model has shortcomings, we feel it represents progress towards finding a high energy completion for the Higgs phase of gravity.
\end{abstract}

DOI: 10.1103/PhysRevD.72.115016

PACS numbers: 12.60.-i, 11.15.Pg, 98.80.Cq

\section{INTRODUCTION}

For a long time physicists hoped that the value of the cosmological constant would be zero, since that might be easier to understand than a small but a nonzero value. But the inference of the existence of dark energy from the supernova data, and its concordance with the cosmic microwave background, gravitational lensing and large-scale structure data, continue to suggest otherwise. These observations give support to the 1987 prediction of Weinberg's that, all else being equal, the cosmological constant cannot be orders of magnitude larger than the cold dark matter component, otherwise galaxies would not have formed [1]. The discovery of only a single new particle at the Large Hadron Collider - the Higgs boson - would also give support to the logical possibility of a landscape [2] in which some of the otherwise seemingly unrelated parameters of the standard model are strongly correlated in order that atoms and galaxies can exist [3].

An alternate - and perhaps testable - possibility is that there is a dynamical explanation for why the cosmological constant is zero. The mechanism responsible for canceling the expected large contributions from standard model particles could perhaps be tested by performing measurements in our own universe. But then there are two remarkable things to explain: what is the dark energy; and why it began to dominate the evolution of the universe only recently. An elegant explanation is provided by tracker [4] versions of quintessence [5]. However, they tend to give the wrong equation of state for the dark energy. Another interesting possibility is that the energy scale of the dark energy is tied to the neutrino mass [6].

The work of [7] rules out an explanation for why the cosmological constant is zero, where the vacuum expectation value of a field relaxes to a value that cancels the "bare" cosmological constant. It has been suggested that topology-changing configurations in Euclidean quantum gravity force the cosmological constant to be zero [8]. See however, [9].

The "ghost condensation" or "Higgs phase of gravity" proposal [10] provides a nontrivial scenario in which the dark energy arises dynamically from a time-dependent scalar field. There has been considerable recent interest in this proposal. See for example [11].

The starting point for ghost condensation is the existence of a scalar with a shift symmetry

$$
\phi \rightarrow \phi+\alpha
$$

that guarantees that the effective action for $\phi$ involves only derivatives acting on $\phi$ :

$$
S=\int d^{4} x \mathcal{L}\left(\partial_{\mu} \phi\right)
$$

Such a theory admits solutions of the form

$$
\phi=c t
$$

where $c$ is a constant. For a free theory in an expanding universe, these solutions become irrelevant at late times. In a nontrivial theory though, they can be important [10].

The effective action that generates such solutions has the form

$$
\mathcal{L}=P(X)
$$

where $X \equiv\left(\partial_{\mu} \phi\right)^{2}$ and we have ignored any operators involving two or more derivatives on $\phi$, since they do not contribute to the equations of motion for solutions of the form (3). However, depending on the form of $P$, in an expanding universe not all of these solutions for $X$ will remain static. The equation of motion for $X$ is

$$
\partial^{\mu}\left(a^{3} P^{\prime}(X) \partial_{\mu} \phi\right)=0
$$


where $a(t)$ is the scale factor. Focusing on solutions that depend only on time,

$$
P^{\prime}(X) \partial_{0} \phi=\frac{\tilde{c}}{a^{3}}
$$

The subsequent evolution of $X$ depends on the form of $P$. If there is a nearby point $X_{*}$ where

$$
P^{\prime}\left(X_{*}\right)=0,
$$

then $X$ will be driven to $X_{*}$ [10]. Such points with $P^{\prime \prime}\left(X_{*}\right)>0$ have the correct sign for the two-time derivative term in the Lagrangian for small fluctuations in $\phi$. Furthermore, the operator $\square(\phi)^{2}$ must be present with a negative coefficient to avoid any spatial instabilities [10]. If $P\left(X_{*}\right)$ is negative, as in Fig. 1, then the dark energy is positive. By contrast, if $P^{\prime}$ has no zeros, say $P \sim X^{n}$ for some range of $X$, then in that interval $P(X) \sim a^{-6 n /(2 n-1)}$ and $P(X)$ becomes increasingly irrelevant at late times.

In the example provided in Fig. 1, the slope near the origin is negative so the theory has a ghost. One might be concerned whether such a ghost could be generated in a theory that at a fundamental level does not have one. It is also possible that there exists an $X_{*}$ where $P^{\prime}\left(X_{*}\right)=0$ but that near the origin the slope is positive. An example of such a $P$ is provided in Fig. 2.

In this paper we investigate whether a renormalizable field theory with a spontaneously broken $U(1)$ symmetry can generate a $P$ of either form for the Goldstone boson, $\phi$. In particular, we will ask whether there can exist solutions with $P^{\prime}=0$ when the underlying field theory is taken to have standard kinetic terms for its fields.

We first consider integrating out the heavy degrees of freedom at the classical level and give a short proof that the spontaneous breaking of a global $U(1)$ symmetry in a general theory of interacting massive scalars does not lead to a $P(X)$ with any extrema. Then we go on to consider a case where the complex scalar, whose vacuum expectation value spontaneously breaks the $U(1)$ symmetry, cou-

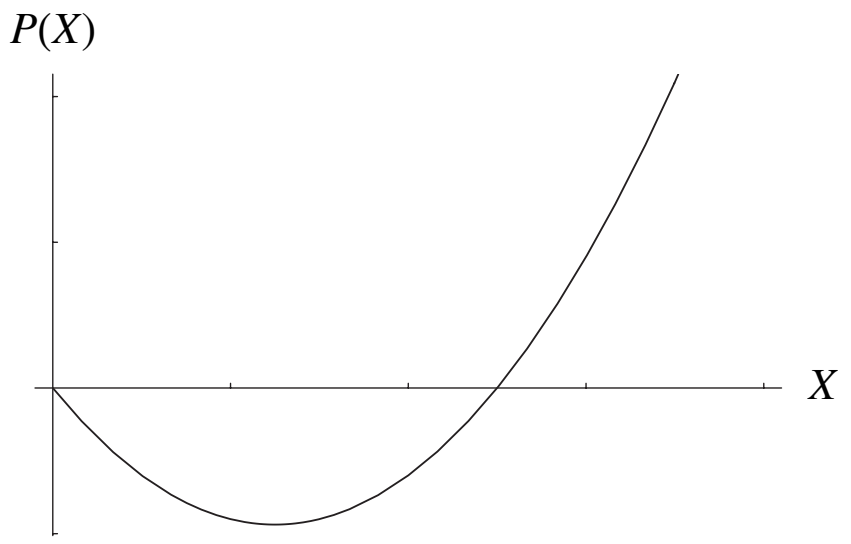

FIG. 1. Nontrivial effective action with asymptotically Lorentz-violating solution.

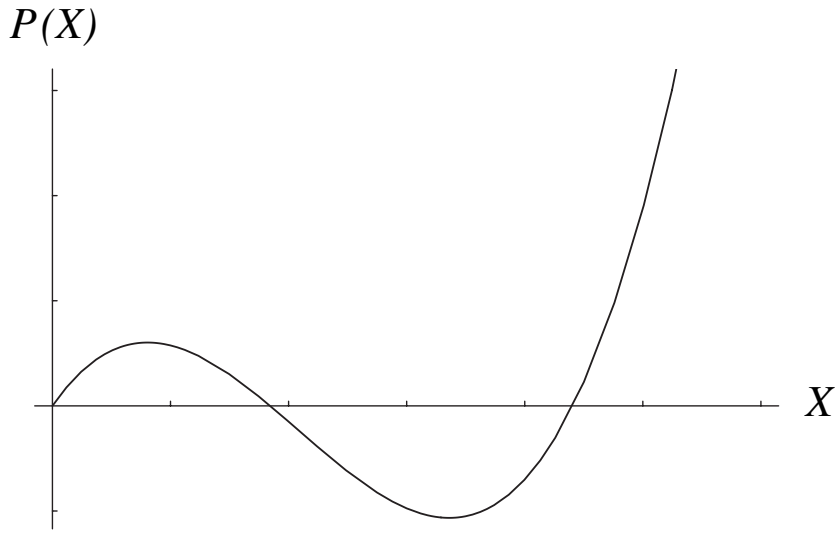

FIG. 2. Another possibility for a nontrivial effective action with an asymptotically Lorentz-violating solution, but no ghost near the origin.

ples to $2 N$ massive fermions. We integrate out the fermions in the large $N$ limit and find that a $P(X)$ for the Goldstone boson like that shown in Fig. 1 can be generated at strong coupling. Even though the theories we are considering are renormalizable, they do require regularization, and hence a cutoff. The model we constructed does not provide a conventional high energy theory for the Higgs phase of gravity because the fermion masses are necessarily near the ultraviolet cutoff of the full theory. Furthermore the low energy theory for the Goldstone boson is unacceptable because it contains a spatial instability. Despite these shortcomings, our work shows that a $P(X)$ like that in Fig. 1 can be generated from an underlying theory with normal kinetic terms.

\section{CLASSICAL PHYSICS}

To start with we consider the very simple example of a single complex field $\Phi$ whose vacuum expectation value (vev) spontaneously breaks a $U(1)$ global symmetry. The renormalizable Lagrangian is

$$
\mathcal{L}_{0}=\partial_{\mu} \Phi^{*} \partial^{\mu} \Phi-\frac{\lambda}{4}\left(|\Phi|^{2}-v^{2}\right)^{2} .
$$

It is convenient to parametrize the fields as

$$
\Phi=\left(v+\frac{b}{\sqrt{2}}\right) e^{i \phi},
$$

where $\phi$ is the Goldstone boson, chosen to not be canonically normalized. $b$ is a massive field that we will integrate out of the theory at the classical level to generate an effective action for the Goldstone boson.

The theory has both a $U(1)$ global symmetry-here realized linearly - and a time translation invariance. The solution

$$
\phi=c t
$$


preserves a linear combination of the time translation symmetry and the nonlinearly realized shift symmetry [10]. We are seeking solutions to the exact equations of motion which respect that symmetry. Inspecting $\Phi$, we note that the magnitude of $\Phi$ though, is invariant only if $b$ is static. This means that we are looking for solutions to the exact classical equations of motion in which $b$ is static and $\phi$ is given by (10).

It is further convenient to define $y \equiv \sqrt{2} b v+\frac{1}{2} b^{2}$. Note that $|\Phi|^{2}=v^{2}+y$ and $y \geq-v^{2} . y=-v^{2}$ is a singular point since there the $U(1)$ is unbroken. In terms of these variables

$$
\mathcal{L}=\left[\frac{1}{2} \frac{\left(\partial_{\mu} y\right)^{2}}{v^{2}+y}+X\left(v^{2}+y\right)-\frac{\lambda}{4} y^{2}\right] .
$$

Next we integrate out $y$ classically, which amounts to solving its classical equations of motion in terms of $X$. As argued above, we assume that there is no time variation in $b$. The solution is then

$$
y=2 \frac{X}{\lambda} .
$$

Substituting this solution for $y$ back into the action gives the effective action for $X$ :

$$
P(X)=X\left(v^{2}+2 \frac{X}{\lambda}\right)-\frac{1}{\lambda} X^{2}=X\left(v^{2}+\frac{X}{\lambda}\right) .
$$

The effective action for $X$ is monotonic and has no extrema. In particular,

$$
P^{\prime}=v^{2}+2 \frac{X}{\lambda}=\left(v^{2}+y[X]\right)=\Phi^{*} \Phi .
$$

Notice that $P^{\prime}(X)$ is positive for all $X$. We now proceed to show that at the classical level this will always be the case.

Consider a general $U(1)$ theory with $N$ scalar fields $\chi_{i}$ each of charge $q_{i}$ (some of which may be zero) and

$$
\mathcal{L}=K-V \text {. }
$$

Here $V$ is the most general potential consistent with the global symmetry (it does not have to be renormalizable.). $K$ is the standard kinetic terms for the scalar fields

$$
K=\sum_{i} \partial_{\mu} \chi_{i}^{*} \partial^{\mu} \chi_{i}
$$

For any value of each $\chi_{i}$, there is a direction in field space that keeps $V$ constant. Promoting this direction, given by

$$
\chi_{i} \rightarrow \chi_{i} e^{i q_{i} \phi},
$$

to a field $\phi$, identifies the Goldstone boson.

To obtain the effective action for $\phi$, we look for solutions that preserve the unbroken combination of time translation and $U(1)$ symmetries, so

$$
\chi_{i}=f_{i}(X) e^{i q_{i} \phi} .
$$

Recall, $X \equiv\left(\partial_{\mu} \phi\right)^{2} . f_{i}$ is static, but may be complex and depend on $X$. The effective action for $f_{i}$ and $X$ is

$$
L=\sum_{i} q_{i}^{2}\left|f_{i}\right|^{2} X-V\left[\left\{f_{j}\right\}\right] .
$$

The equation of motion for $f_{i}^{*}$ gives

$$
q_{i}^{2} X f_{i}=\frac{\partial V}{\partial f_{i}^{*}} .
$$

Solving the $N$ equations determines $f_{i}(X)$. The effective action for $X$ is then

$$
P(X)=\sum_{i} q_{i}^{2} X\left|f_{i}(X)\right|^{2}-V\left[\left\{f_{j}(X)\right\}\right]
$$

so that

$$
\begin{aligned}
P^{\prime}(X)= & \sum_{i} q_{i}^{2}\left|f_{i}\right|^{2}+\sum_{i} q_{i}^{2} X\left[f_{i}^{*} \frac{\partial f_{i}}{\partial X}+\text { h.c. }\right] \\
& -\sum_{i}\left[\frac{\partial V}{\partial f_{i}} \frac{\partial f_{i}}{\partial X}+\text { h.c. }\right] \\
= & \sum_{i} q_{i}^{2}\left|f_{i}\right|^{2} \geq 0 .
\end{aligned}
$$

In this class of theories, $P$ is monotonically increasing. From this expression we see that $P$ can have an extrema only if all the fields vanish: $f_{i}=0$. At this location the $U(1)$ global symmetry is unbroken and there is no Goldstone boson. Note that,

$$
P^{\prime}(X)=\left.\frac{\partial K\left[\left\{f_{i}\right\}, X\right]}{\partial X}\right|_{f_{i}=f_{i}(X)} .
$$

(23) is also valid for any $K$ that is analytic in $\partial_{\mu} \chi_{i}^{*} \partial^{\mu} \chi_{i}$.

Equation (22) reproduces the previous result for $P^{\prime}$ in the one field model considered earlier, where, $q=1$ and $|f|^{2}=|\Phi|^{2}=v^{2}+y$.

\section{TOWARDS A HIGH ENERGY COMPLETION}

The toy model we consider has a complex scalar $\Phi$ and two Dirac multiplets of fermions $\psi_{1}$ and $\psi_{2}$. Under the $U(1)$ global symmetry, the scalar has charge +1 and the fermions have charge +1 and +2 respectively. The model also has a global $S U(N)$ symmetry, where the fermions each transform under the fundamental representation and the scalar is neutral. We suppress the $S U(N)$ indices on the fermions.

The most general renormalizable Lagrangian, consistent with these symmetries, is

$$
\begin{aligned}
\mathcal{L}= & \partial_{\mu} \Phi^{*} \partial^{\mu} \Phi-\frac{\lambda}{4}\left(|\Phi|^{2}-v^{2}\right)^{2}+\sum_{i=1,2}\left(i \bar{\psi}_{i} \gamma^{\mu} \partial_{\mu} \psi_{i}\right. \\
& \left.-m_{\psi} \bar{\psi}_{i} \psi_{i}\right)-g \Phi \bar{\psi}_{2} \psi_{1}-g \Phi^{*} \bar{\psi}_{1} \psi_{2} .
\end{aligned}
$$

Note that all the fields have the conventional sign for their kinetic terms. We have performed a field redefinition to make $g$ real and for simplicity have taken the two types of fermions to have equal masses $m_{\psi}$. 
Loops involving the fermions and scalar selfinteractions generate an effective action for $\Phi$. To generate a ghostlike kinetic term for $\Phi$, quantum corrections must overcome the tree-level kinetic term. This implies that perturbation theory is not valid. Hence we need the exact effective action to conclude that quantum corrections have flipped the sign of the kinetic term for $\Phi$. The large quantum corrections come from fermion loops. To have control over these, we consider the limit $N$ large with $g^{2} N$ fixed. Corrections from the scalar self-interactions will be treated classically, so we assume that $\lambda$ is small.

In the large $N$ limit the only loop diagram that contributes to the effective action for $\Phi$ is given by Fig. 3 and is proportional to $g^{2} N$. All other diagrams are suppressed by powers of $1 / N$, see for example [12]. (Actually, at leading order there is also a two-loop diagram which contributes to the cosmological constant).

To compute the effective action requires regularization and renormalization of the parameters in the theory. We use dimensional regularization and subtract using the $\overline{\mathrm{MS}}$ scheme. In the large $N$ limit there is mass and wave function renormalization for $\Phi$, but none for either fermion. The only renormalization of the coupling $g$ is due to the wave function renormalization of $\Phi$. Denoting bare quantities with a subscript " 0 " and those without as the renormalized quantities, one has

$$
\begin{array}{lc}
\Phi_{0}^{*} \Phi_{0}=Z_{\Phi} \Phi^{*} \Phi & g_{0}^{2} Z_{\Phi}=g^{2} \mu^{\epsilon} \\
\lambda_{0} Z_{\Phi}^{2}=\lambda \mu^{\epsilon} & m_{\Phi, 0}^{2} Z_{\Phi}=m_{\Phi}^{2}+\delta m^{2}
\end{array}
$$

with the renormalized quantities implicit functions of the subtraction scale $\mu$ and $d=4-\epsilon$. Then

$$
\begin{gathered}
Z_{\Phi}=1-\frac{g^{2} N}{8 \pi^{2}}\left(\frac{2}{\epsilon}-\gamma_{E}+\ln (4 \pi)\right) \\
\delta m^{2}=-\frac{3 g^{2} N}{4 \pi^{2}} m_{\psi}^{2}\left(\frac{2}{\epsilon}-\gamma_{E}+\ln (4 \pi)\right) .
\end{gathered}
$$

One finds the $\beta$ function

$$
\beta(g)=-\frac{\epsilon}{2} g Z_{\Phi}
$$

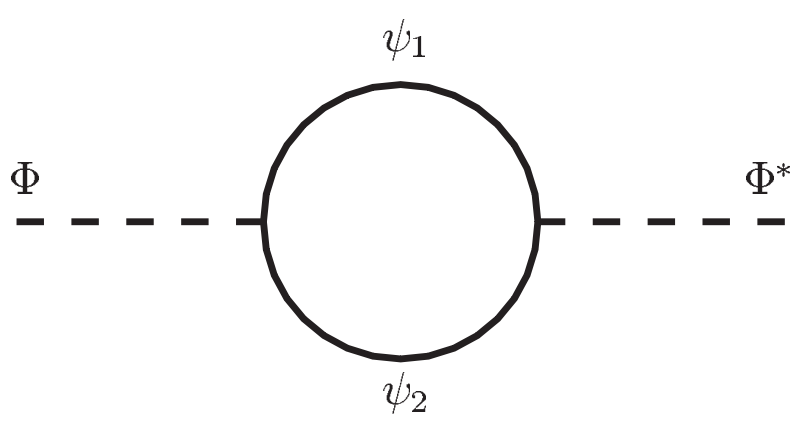

FIG. 3. Leading one-loop diagram contributing to effective action for $\Phi$.
In the limit $\epsilon \rightarrow 0$, one obtains the exact solution

$$
\frac{1}{g^{2}(\mu)}=\frac{1}{g^{2}\left(\mu_{0}\right)}-\frac{N}{4 \pi^{2}} \ln \left[\frac{\mu}{\mu_{0}}\right] .
$$

As expected, the theory has a Landau pole. One notes that since there is only wave function renormalization of the coupling $g, g^{2} \Phi^{*} \Phi$ is independent of $\mu$.

In terms of the renormalized quantities, the full effective action for $\Phi$ is

$$
\int d^{4} x \mathcal{L}_{\mathrm{eff}}=\int d^{4} p \tilde{\Phi}^{*}(-p) G\left(p^{2}\right) \tilde{\Phi}(p)-\int d^{4} x V(|\Phi|)
$$

where

$$
\begin{gathered}
V=m_{\Phi}^{2}(\mu) \Phi^{*} \Phi+\frac{\lambda(\mu)}{4}\left(\Phi^{*} \Phi\right)^{2}, \\
G\left(p^{2}\right)=g^{2}(\mu)\left\{\frac{p^{2}}{g^{2}(\mu)}-\frac{N p^{2}}{4 \pi^{2}} \ln \left[\frac{m_{\psi}}{\mu}\right]\right. \\
+\frac{N}{4 \pi^{2}}\left(2 m_{\psi}^{2}-\frac{1}{2} p^{2}\right) \int_{0}^{1} d y \ln \left[1-y(1-y) \frac{p^{2}}{m_{\psi}^{2}}\right] \\
\left.-\frac{N}{4 \pi^{2}} m_{\psi}^{2}\left(1-6 \ln \left[\frac{m_{\psi}}{\mu}\right]\right)\right\}
\end{gathered}
$$

and $\tilde{\Phi}(p)$ denotes the Fourier transform of $\Phi(x)$. Since the effective action is exact, it should be independent of $\mu$. Inspecting the above equation, there are several sources for such a dependence. From the running of $g$, one sees that the $\mu$-dependence of the first two terms in $G$ cancel. In fact, together they combine into $p^{2} / g^{2}\left(m_{\psi}\right)$. The other sources in $G$ of $\mu$-dependence is the over all dependence on $g^{2}(\mu)$, and in the term in the last line. The overall $\mu$ dependence proportional to $g^{2}(\mu)$ cancels the wave function renormalization of $\Phi$. The $\mu$ dependence of the last term is canceled by the $\mu$ dependence of the $m_{\Phi}^{2}$ term in the potential. Likewise, the other terms in the potential are $\mu$ independent. After shifting $G(0)$ into the mass term, $\lambda$ and $m_{\Phi}^{2}$ run only by wave function renormalization.

The effective action has a cut beginning at the branch point $p^{2}=4 m_{\psi}^{2}$ corresponding to fermion pair production. For $p^{2}$ less than this value we can power series expand in $p^{2} / m_{\psi}^{2}$, transform to position space, then resum to get

$$
\mathcal{L}_{\text {eff }}=\Phi^{*} G(-\square) \Phi-V(|\Phi|) .
$$

Note that if the magnitude of $\Phi$ is frozen, then, $-\square \rightarrow X$. The integral over the Feynman parameter $y$ can be done exactly for $p^{2}<4 m_{\psi}^{2}$. One gets

$$
G(-\square)=g^{2}(\mu)\left(\frac{-\square}{g^{2}\left(m_{\psi}\right)}+\frac{N}{4 \pi^{2}} m_{\psi}^{2} f\left(-\square / m_{\psi}^{2}\right)\right)
$$

with 


$$
f(z)=(4-z)\left[-1+\frac{\sqrt{4-z}}{\sqrt{z}} \arctan \left(\frac{\sqrt{z}}{\sqrt{4-z}}\right)\right] .
$$

Note that we have shifted $G(0)$ into the renormalized mass $m_{\Phi}^{2}$ so that in (34) $G(0)=0$.

We now expand the effective action in powers of $\square / m_{\psi}^{2}$. For small $z$

$$
f(z)=-\frac{z}{3}+\frac{z^{2}}{20}+\frac{z^{3}}{280}+\frac{z^{4}}{2520}+\cdots .
$$

Then

$$
G(-\square)=g^{2}(\mu)\left(\frac{1}{g^{2}\left(m_{\psi}\right)}-\frac{N}{12 \pi^{2}}\right)(-\square)+\mathcal{O}\left(\frac{\square^{2}}{m_{\psi}^{2}}\right) .
$$

Since the effective Lagrangian is independent of $\mu$ (the running of $g^{2}$ is canceled by the wave function renormalization) we are free to choose $\mu=m_{\psi}$. Introducing

$$
\gamma=\frac{g^{2}\left(m_{\psi}\right) N}{12 \pi^{2}}-1
$$

the effective action for $\Phi$ at scales much below $m_{\psi}$ is

$$
\mathcal{L}_{\text {eff }}=-\gamma \partial^{\mu} \Phi^{*} \partial_{\mu} \Phi-V\left(\Phi^{*} \Phi\right) .
$$

Terms suppressed by powers of $1 / m_{\psi}^{2}$ have been dropped.

The important observation at this stage is that for large enough coupling $g^{2}\left(m_{\psi}\right) N$, i.e. $\gamma>0$, the field $\Phi$ has a kinetic term with the wrong sign. This is one of our main results. Quantum corrections from the fermions have generated a wrong-signed kinetic term. However, the large coupling needed implies the fermion masses are near the Landau pole. Nonetheless, our conclusions do not depend on the use of dimensional regularization. In the appendix we consider a general class of translation invariant regulators that cutoff the momentum on the order of $\Lambda$. For large enough bare coupling $g_{0}^{2} N$ and by having the fermion masses $m_{\psi}$ of order $\Lambda$, a wrong-signed kinetic term can be generated.

If $m_{\psi}$ is not treated as large, the model (33) does lead to a minimum for $P(X)$. However, the dynamics of $X$ [encoded in $P(X)$ ] cannot be separated from the fermion mass $m_{\psi}$, and hence the cutoff. We therefore take the limit $m_{\psi}$ large and use the results of section II, where it was shown that classically integrating out scalars coupled to $\Phi$ will always generate a correction to $P(X)$ that is monotonically increasing. That will generate a $P(X)$ of the form appearing in Fig. 1, where the location of the minimum is now at a scale much below the ultraviolet cutoff.

To illustrate how that can be done, we choose the following simple model. We add a scalar $S$, also of charge +1 , and only consider mass mixing between $\Phi$ and $S$. Thus to the Lagrange density (24) we add

$$
\delta \mathcal{L}=\partial^{\mu} S^{*} \partial_{\mu} S-m_{S}^{2} S^{*} S-h^{2} S^{*} \Phi-h^{2} S \Phi^{*}
$$

where we have made a phase redefinition on $S$ so that $h$ is real and positive and further assume that $h<m_{S}$. This model is not realistic, because other interactions consistent with the symmetries of the theory have not been included. But the point here is just to illustrate that a toy model exists which can generate a $P(X)$ with a local minimum.

Solving for $S$ at the classical level gives

$$
S=-h^{2} \frac{1}{\square+m_{S}^{2}} \Phi
$$

leading to

$$
\mathcal{L}_{\text {eff }}=\Phi^{*}\left(\gamma \square+h^{4}\left[\frac{1}{\square+m_{S}^{2}}-\frac{1}{m_{S}^{2}}\right]\right) \Phi-V\left(\Phi^{*} \Phi\right)
$$

where we have shifted a mass squared term for $\Phi$ into the potential. The kinetic term for $\Phi$ can be rewritten as

$$
\Phi^{*}\left(\left[\gamma-\frac{h^{4}}{m_{S}^{4}}\right] \square+\frac{h^{4}}{m_{S}^{4}} \frac{\square^{2}}{\square+m_{S}^{2}}\right) \Phi .
$$

Note that by choosing the coupling $g^{2} N$ large enough and $\gamma>h^{4} / m_{S}^{4}$, one can maintain a wrong-signed kinetic term for $\Phi$. Defining

$$
\epsilon^{\prime}=\gamma-\frac{h^{4}}{m_{S}^{4}}>0,
$$

the effective action for $\Phi$ is of the form

$$
\mathcal{L}_{\text {eff }}=\Phi^{*} F(-\square) \Phi-V\left(\Phi^{*} \Phi\right)
$$

where

$$
F(z)=-\epsilon^{\prime} z+\frac{h^{4}}{m_{S}^{4}} \frac{z^{2}}{m_{S}^{2}-z} .
$$

For $z<m_{S}^{2}$ the second term is a monotonically increasing function of $z$.

Next we look for solutions to the exact effective action of the form

$$
\Phi=\sigma e^{i \phi}
$$

where $\phi=c t$. We will assume that in this background $\langle\sigma\rangle$ is nonzero and constant, so that the $U(1)$ symmetry is broken.

For solutions with both $X$ and $\sigma$ constant, the effective action is

$$
\mathcal{L}_{\text {eff }}=\sigma^{2} F(X)-m_{\Phi}^{2} \sigma^{2}-\frac{\lambda}{4} \sigma^{4} .
$$

We obtain the effective action for $X$ by solving for $\sigma$ and integrating it out. One finds that

$$
\sigma^{2}=\frac{2}{\lambda}\left(F(X)-m_{\Phi}^{2}\right)
$$

and we assume that $-m_{\Phi}^{2}+F(X)>0$. In terms of $v^{2}=$ $-2 m_{\Phi}^{2} / \lambda$, the vev of the potential at $X=0$, one finds 


$$
P(X)=v^{2} F(X)+\frac{F(X)^{2}}{\lambda} .
$$

Then

$$
P^{\prime}(X)=F^{\prime}(X) v^{2}\left(1+\frac{2 F(X)}{\lambda v^{2}}\right)=F^{\prime}(X) \sigma^{2} .
$$

Thus the sign and zeroes of $P^{\prime}$ are the same as for $F^{\prime}$. In particular, with $\epsilon^{\prime}>0, P^{\prime}(X=0)<0$.

To determine the minimum of $P$ and study its stability, it is convenient to assume $\epsilon^{\prime} \ll 1$. This separates the scale $\langle X\rangle$ from the scale of $m_{S}$ or $v$, thus simplifying the analysis. Then

$$
F(X)=-\epsilon^{\prime} X+\frac{h^{4}}{m_{S}^{4}} \frac{X^{2}}{m_{S}^{2}}+\mathcal{O}\left(\frac{X^{3}}{m_{S}^{4}}\right)
$$

has a local minimum at

$$
X=\frac{1}{2 \gamma} \epsilon^{\prime} m_{S}^{2}\left(1+\mathcal{O}\left(\epsilon^{\prime}\right)\right)
$$

So with $\epsilon^{\prime} \ll \gamma \simeq h^{4} / m_{S}^{4}<1,\langle X\rangle$ is much less than $m_{S}$.

Inspecting the expression for $P$ in (50), one notes that the second term of $\mathcal{O}\left(F^{2}\right)$ is subdominant to the first contribution in the limit $\epsilon^{12} m_{S}^{2} \ll \gamma \lambda v^{2}$. In this limit the effective action for $X$ simplifies to

$$
P(X) \simeq F(X) v^{2},
$$

and the minimum for $X$ given by (53) is a local minimum of $P$. Using (46) and (50), a graph of $P(X)$ is given in Fig. 4 for $\epsilon^{\prime}=0.01, m_{S}=1, \lambda v^{2} / m_{S}^{2}=1 / 9$ and $h^{4} / m_{S}^{4}=1 / 10$. $P(X)$ is given in units of $m_{S}^{2} v^{2}$. The dark energy arising from the time-dependence of $\phi$ is of order $\epsilon^{\prime 2} v^{2} m_{S}^{2} / \gamma$. Of course, in this toy model there is in addition a contribution to the cosmological constant from the potential which must be canceled.

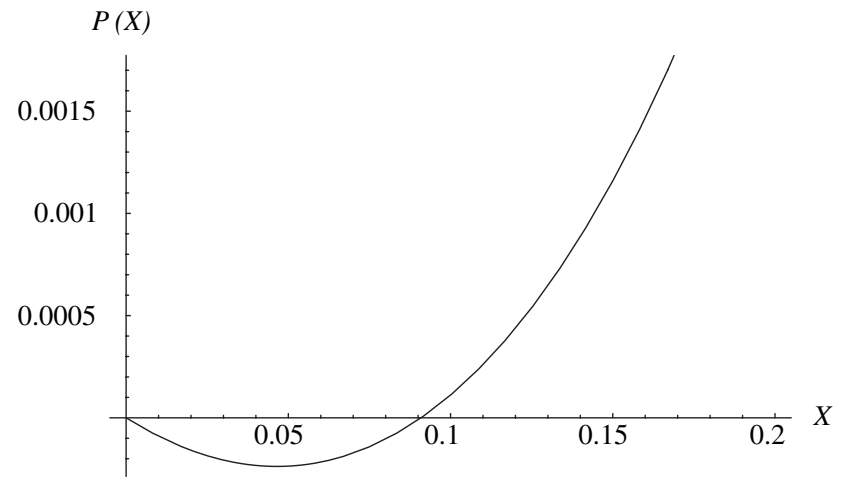

FIG. 4. $\quad P(X)$ for $\epsilon^{\prime}=0.01, m_{S}=1, \lambda v^{2} / m_{S}^{2}=1 / 9$ and $h^{4} / m_{S}^{4}=1 / 10 . P(X)$ is in units of $m_{S}^{2} v^{2}$.

\section{STABILITY ANALYSIS}

In this section we study the stability of our theory. Since in an expanding universe $X$ is driven to $P^{\prime}(X)=0$ at late times [10], we focus on the dynamics at that point.

The model has two fluctuating degrees of freedom, $\hat{\phi}$ and $\hat{\sigma}$ which are defined by expanding about the background expectation value of $\Phi$,

$$
\phi=c t+\hat{\phi}, \quad \sigma=\sigma_{0}+\hat{\sigma}
$$

where $\Phi=\left(\sigma_{0}+\hat{\sigma}\right) e^{i c t+i \hat{\phi}}$. It is convenient to introduce $c_{\mu} \equiv c \delta_{\mu 0}$ and recall that $X=c_{\mu} c^{\mu}$. As discussed in the previous section, $c_{\mu}$ and $\sigma_{0}$ are given by solving the classical equations of motion. We assume the parameters of the model are such that $\sigma_{0} \neq 0$ and restrict our attention to terms with up to two-time derivatives acting on the fields.

We begin by studying the dynamics of $\hat{\sigma}$. About the minimum of the potential it will have a positive mass squared. Its dependence on $X$ and the parameters of the model is not needed here. For the stability analysis, it is sufficient to determine terms in the effective action involving only two $\hat{\sigma}$ fields. Defining $D_{\mu} \equiv \partial_{\mu}+i c_{\mu}$, the action to quadratic order in $\hat{\sigma}$ is given by

$$
\mathcal{L}_{\text {eff }}(\sigma)=\sigma F\left(-D^{2}\right) \sigma-\frac{\lambda}{4}\left(\sigma^{2}-v^{2}\right)^{2} .
$$

With $D^{2}=\partial^{2}+2 i c \cdot \partial-c^{2}$, expanding the action about $D^{2}=-c^{2}$ to the four-spatial derivative and two-time derivative levels gives, for the terms involving derivatives only,

$$
\mathcal{L}_{\text {eff }}(\sigma)=F^{\prime \prime}\left(c^{2}\right) \hat{\sigma}\left[-2(c \cdot \partial)^{2}+\frac{1}{2} \square^{2}\right] \hat{\sigma}+\cdots .
$$

We note that if $F^{\prime \prime}\left(c^{2}\right)>0$ then $\hat{\sigma}$ has a healthy two-time derivative term.

The effective Lagrangian for $\hat{\sigma}$ has no two-gradient terms. The leading gradient terms then come from the second term involving four spatial derivatives and is

$$
\frac{1}{2} F^{\prime \prime}\left(c^{2}\right) \hat{\sigma} \nabla^{4} \hat{\sigma} \text {. }
$$

Since for stability of the kinetic energy (i.e. two-time derivative term) we require that $F^{\prime \prime}>0$, the four-gradient term has the wrong sign. The dispersion relation for $\hat{\sigma}$ is then $4 c^{2} F^{\prime \prime}\left(c^{2}\right) E^{2}=m_{\sigma}^{2}-F^{\prime \prime}\left(c^{2}\right) k^{4}$. The instability only occurs at short wavelengths of order $k^{4} \simeq m_{\sigma}^{2} / F^{\prime \prime}\left(c^{2}\right)$.

In this model the stability of the kinetic energy and the gradient energy of $\hat{\sigma}$ place opposing requirements on the curvature of $F$. Fortunately it is not difficult to enlarge the model generating the correct sign for $\hat{\sigma}$ 's two-gradient term. For example, consider a massive real scalar $S^{\prime}$ neutral under the $U(1)$ symmetry and the following Lagrangian

$$
\mathcal{L}_{S}=-\frac{1}{2} S^{\prime} \square S^{\prime}-\frac{1}{2} m_{S^{\prime}}^{2} S^{\prime 2}+g^{\prime} S^{\prime} \Phi^{*} \Phi .
$$


By integrating out $S^{\prime}$ at the tree-level we obtain the following effective Lagrangian

$$
\delta \mathcal{L}_{\text {eff }}=\frac{g^{\prime 2}}{2 m_{S^{\prime}}^{2}} \Phi^{*} \Phi \frac{1}{1+\square / m_{S^{\prime}}^{2}} \Phi^{*} \Phi
$$

which gives a healthy kinetic term to the radial mode at the two-derivative level,

$$
\delta \mathcal{L}_{\text {eff }}=-\frac{g^{\prime 2}}{2 m_{S^{\prime}}^{4}} \Phi^{*} \Phi \square\left(\Phi \Phi^{*}\right) \rightarrow-\frac{2 g^{\prime 2} \sigma_{0}^{2}}{m_{S^{\prime}}^{4}} \hat{\sigma} \square \hat{\sigma} .
$$

We assume that the coefficient of this contribution is large enough to stabilize $\hat{\sigma}$. Note that since $S^{\prime}$ is neutral, the above modification to the effective action for $\Phi$ is independent of the Goldstone boson.

Next we turn to the stability condition for the Goldstone field $\hat{\phi}$. As before, we investigate the dynamics at $P^{\prime}=$ $F^{\prime}=0$. Since we have stabilized $\hat{\sigma}$ in the preceding discussion, we ignore its fluctuations in discussing the stability of the Goldstone boson. ${ }^{1}$ Then the effective action for $\hat{\phi}$ is

$$
\mathcal{L}_{\mathrm{eff}}=\sigma_{0}^{2} e^{-i \hat{\phi}} F\left(-D^{2}\right) e^{i \hat{\phi}} .
$$

The part of the effective action involving two $\hat{\phi}$ fields is

$$
\mathcal{L}_{\text {eff }}=\frac{1}{2} F^{\prime \prime}\left(c^{2}\right)\left[\hat{\phi} \square^{2} \hat{\phi}-4 \hat{\phi}(c \cdot \partial)^{2} \hat{\phi}\right]+\cdots .
$$

Here $\hat{\phi}$ has the correct sign for its two-time derivative kinetic term if $P^{\prime \prime}=F^{\prime \prime} \sigma_{0}^{2}>0$. As in [10], its twoderivative gradient term vanishes. Unfortunately, $\hat{\phi}$ has the wrong sign for its four-derivative gradient term since $P^{\prime \prime}>0$. This leads to the dispersion relation (neglecting terms involving $E^{4}$ and $\left.k^{2} E^{2}\right) E^{2} \simeq-k^{4} / 4 c^{2}$, which has instabilities at small $k$.

These spatial instabilities can be removed at the expense of adding higher dimensional operators. One possibility is to add

$$
\mathcal{L}_{\hat{\phi}}=+\frac{\lambda^{\prime}}{2}\left(\Phi^{*} \square \Phi\right)^{2}+\text { h.c., }
$$

with $\lambda^{\prime}>0$. This operator contributes $\delta P(X)=\lambda^{\prime} X^{2} \sigma_{0}^{4}$, shifting the minimum of $P(X)$ and $\sigma_{0}$, but not affecting $P^{\prime}(X=0)<0$. Expanding this operator (about the new minimum) to quadratic order in small fluctuations for $\hat{\phi}$, one finds

$$
\delta \mathcal{L}_{\hat{\phi}}=\lambda^{\prime} \sigma_{0}^{4}\left(-\hat{\phi} \square^{2} \hat{\phi}-4 \hat{\phi}(c \cdot \partial)^{2} \hat{\phi}\right)
$$

where we have dropped terms with more derivatives. Note

\footnotetext{
${ }^{1}$ Properly integrating out $\sigma$ does not change this conclusion. From solving the classical equations of motion at the point $P^{\prime}\left(c^{2}\right)=0$, one finds an additional contribution to $\sigma$ involving spatial gradients that is of the form $\delta \sigma \propto F^{\prime \prime} \sigma_{0}\left[e^{-i \hat{\phi}} \nabla^{4} e^{i \hat{\phi}}+\right.$ h.c.]. The point is that since $\delta \sigma \sim \nabla^{4} \hat{\phi}$, inserting it back into the action generates terms for $\hat{\phi}$ involving six spatial derivatives.
}

that with $\lambda^{\prime}>0$, this operator gives the correct sign to terms involving four spatial derivatives and to terms involving two time derivatives acting on $\hat{\phi}$. Thus when added to the Lagrangian with large enough coefficient, it can remove the instability in the four-gradient term without affecting the requirement that the kinetic energy term involving two time derivatives is positive or the behavior of $P^{\prime}(X=0)$. Although the operator in (65) is not renormalizable, it may be possible to generate it by integrating out heavy degrees of freedom.

\section{CONCLUSIONS}

The Higgs phase of gravity or ghost condensation is an interesting proposal for the dark energy. There the dark energy arises dynamically from a time-dependent scalar field $\phi$ that spontaneously breaks Lorentz invariance. The effective action for the $\phi$ contains only derivatives of the field so it is natural to hypothesize that $\phi$ is the Goldstone boson resulting from a spontaneously broken $U(1)$ symmetry. It is conventional to introduce the notation $X=$ $\left(\partial_{\mu} \phi\right)^{2}$ and use $P(X)$ to denote the part of the Lagrangian that contains single derivatives on $\phi$. Usually the evolution of the universe redshifts away the energy density stored in the time dependence of $\phi$. However, if there is a value of $X$ where $P^{\prime}(X)=0$ then Lorentz symmetry is broken asymptotically as $t \rightarrow \infty$ and the time dependent scalar field is a candidate for the dark energy. In Figs. 1 and 2 examples of such Lagrange densities are shown. In the first case $P^{\prime}(0)<0$ which corresponds to a wrong sign kinetic term for $\phi$.

In this paper we studied the possibility that the Higgs phase of gravity is the low energy limit of an underlying theory that is renormalizable, has standard kinetic terms for its fields and spontaneously breaks a $U(1)$ symmetry. In particular we are interested in whether a $P(X)$ of the form shown in Fig. 1 or Fig. 2 can be generated by integrating out the heavy degrees of freedom in such models.

Our results show that in a wide class of theories involving only scalars, the spontaneous breaking a global $U(1)$ symmetry leads, at the classical level, to an effective action $P(X)$ for the Goldstone boson that is always monotonically increasing. While such an effective action can have solutions that break Lorentz invariance, those solutions are not relevant at late times in an expanding Friedman-RobertsonWalker universe.

We examined a model where the field whose vev spontaneously breaks the global $U(1)$ symmetry is coupled to $2 N$ massive fermions and another complex scalar. In the large $N$ limit the effective action for the scalar field that directly couples to the fermions can be computed exactly. At large enough $g^{2} N$, an effective action of the form displayed in Fig. 1 is obtained.

Unfortunately this model is not a satisfactory high energy theory for two reasons. The theories we consider are renormalizable but still require regularization via an ultra- 
violet cutoff. Unlike in conventional theories, here we find that the fermion masses cannot be taken to be small compared with the cutoff. ${ }^{2}$ Second, there is a spatial instability for the Goldstone boson. By extending the model considered in this paper, it may be possible to overcome the latter difficulty. Our work is progress towards the goal of finding high energy completions for low energy effective theories with ghost condensation.

\section{ACKNOWLEDGMENTS}

This work was supported by grants from the Department of Energy under Grant Nos. DE-FG02-90ER40542 at IAS and DE-FG03-92ER40701 at Caltech. I. L. would like to thank the theory group at Caltech for hospitality during the completion of this work.

\section{APPENDIX}

Since in the model of Sec. III the fermion masses were of order the Landau pole, one might worry that the conclusions of that section depend on the use of dimensional regularization. In this Appendix we compute the effective action for $\Phi$ using the Lagrangian in (24) but regulate the theory with a momentum cutoff rather than with dimensional regularization. We shall see that for a range of values for $m_{\psi}$ of order $\Lambda$ and large enough bare coupling $g_{0}^{2} N$, quantum corrections to the effective action can generate a wrong-signed kinetic term for $\Phi$.

As before, at large $N$ the Feynman diagram in Fig. 3 is the exact quantum correction to the effective action for $\Phi$. To regulate the diagram we first Wick rotate both the external and internal momenta into Euclidean space. At the end of the computation, we Wick rotate the external momentum back to Minkowski signature. To preserve translation invariance, we regulate the Euclidean space propagators by modifying them accordingly [13],

$$
\frac{1}{(k+p)^{2}+m_{\psi}^{2}} \rightarrow \frac{1}{(k+p)^{2}+m_{\psi}^{2}} \mathcal{K}\left[(k+p)^{2} / \Lambda^{2}\right]
$$

where $\mathcal{K}[x] \rightarrow 0$ for large $x$. To begin with, the regulator we consider is

$$
\mathcal{K}\left[q^{2}\right]=e^{-q^{2} / \Lambda^{2}} .
$$

In (Euclidean) position space this is equivalent to introducing a term $e^{-\square / \Lambda^{2}}$ into the kernel. To extract the $p^{2}$ coefficient, we expand all terms to that order. Focusing on the $p^{2}$ terms only gives

$$
\mathcal{L}_{\text {eff }}=\Phi^{*} F(-\square) \Phi
$$

\footnotetext{
${ }^{2}$ We considered two different regulators, a general translation invariant momentum cutoff satisfying very reasonable assumptions, and dimensional regularization. Our conclusions do not depend on the explicit choice of regulator.
}

(now $\square$ is in Minkowski space) where

$$
F(z)=z+\frac{g_{0}^{2} N}{4 \pi^{2}} z h(w)
$$

with

$$
\begin{aligned}
h(w)= & \int_{0}^{\infty} d x e^{-2 x / w} \frac{x}{(x+1)^{2}}\left(( 1 - x ) \left[-\frac{1}{w}-\frac{1}{x+1}\right.\right. \\
& \left.\left.+\frac{x}{(x+1)^{2}}+\frac{1}{2} \frac{x}{w^{2}}+\frac{x}{x+1} \frac{1}{w}\right]+\frac{1}{2} \frac{x}{x+1}+\frac{1}{2} \frac{x}{w}\right)
\end{aligned}
$$

and $w \equiv \Lambda^{2} / m_{\psi}^{2}$. The first term appearing in (A4) is the tree result. By inspecting (A5) in the limit $w \ll 1$ one can see without much work that $h(w)<0$ for small $w$. A plot of $h(w)$ is shown in Fig. 5 for a larger range of $w$. Note that for $0 \leq \Lambda^{2} / m_{\psi}^{2} \leq 1.1$ the function $h$ is negative.

Recalling the result from Sec. II that

$$
P^{\prime}(X)=\frac{\partial F}{\partial X} \sigma^{2}
$$

evaluated at $\sigma(X)$,

$$
P^{\prime}(X=0)=\sigma^{2}\left[1+\frac{g_{0}^{2} N}{4 \pi^{2}} h\left(\frac{\Lambda^{2}}{m_{\psi}^{2}}\right)\right] .
$$

Inspecting the result (A7) for $P^{\prime}(X=0)$, we see that since there is a range for $\Lambda^{2} / m_{\psi}^{2}$ for which $h<0$, then for each $\Lambda / m_{\psi}$ there exists a critical coupling $g_{\text {crit }}^{2} N$ such that for $g_{0}^{2} N>g_{\text {crit }}^{2} N, P^{\prime}(X=0)<0$.

Next we take the limit that both $\Lambda$ and $m_{\psi}$ are both very large (say of the order the grand unification scale), but holding $w=\Lambda^{2} / m_{\psi}^{2}$ fixed at a value for which $h(w)<0$. We also choose $g_{0}^{2} N>g_{\text {crit }}^{2} N$. Then the effective theory for $\Phi$ far below the cutoff is

$$
\mathcal{L}_{\text {eff }}=-\gamma \partial^{\mu} \Phi^{*} \partial_{\mu} \Phi-V\left(\Phi^{*} \Phi\right),
$$

where $\gamma=-1-g_{0}^{2} N h\left(\Lambda^{2} / m_{\psi}^{2}\right) / 4 \pi^{2}>0$. Higher order terms are suppressed by $p^{2} / \Lambda^{2}$ and are irrelevant.

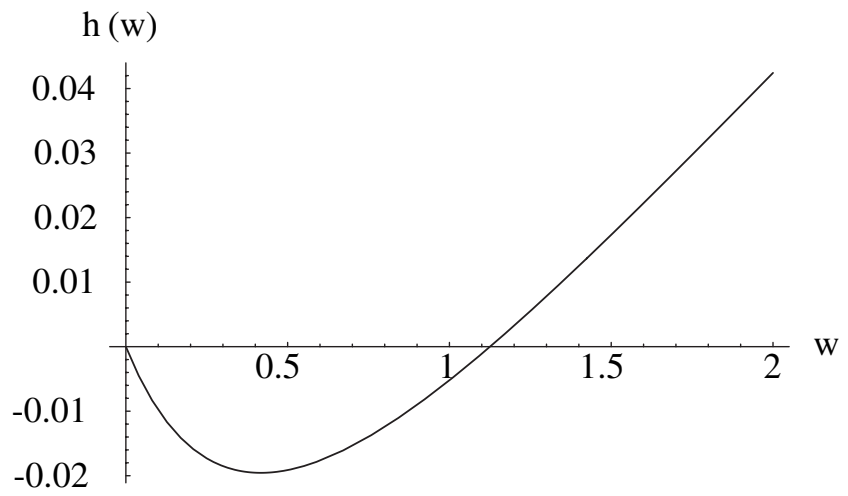

FIG. 5. $h(w)$. Note that $h<0$ for a range of $w=\Lambda^{2} / m_{\psi}^{2}$. The growth for large $w$ is logarithmic. 
The above results were obtained using the exponential regulator (A2). The conclusion that $h<0$ for $\Lambda^{2} \ll m_{\psi}^{2}$ is true, however, for a general $\mathcal{K}$. To see that, note that in the limit $\Lambda^{2} \ll m_{\psi}^{2}$, the $p$-dependence of the two-point function is dominated by the factor $\mathcal{K}\left[(k+p)^{2} / \Lambda^{2}\right]$ in the loop integral. The dominant contribution to $h$ in this limit is then

$$
\begin{aligned}
h\left(\frac{\Lambda^{2}}{m_{\psi}^{2}}\right) & =\frac{m_{\psi}^{2}}{\Lambda^{2}} \int_{0}^{\infty} d x x \mathcal{K}[x]\left(\mathcal{K}^{\prime}[x]+\frac{1}{2} x \mathcal{K}^{\prime \prime}[x]\right) \\
& =-\frac{m_{\psi}^{2}}{2 \Lambda^{2}} \int_{0}^{\infty} d x x^{2} \mathcal{K}^{\prime 2}<0 .
\end{aligned}
$$

To arrive at the second line we have integrated by parts and assumed that $x^{2} \mathcal{K} \mathcal{K}^{\prime}(x) \rightarrow 0$ as $x \rightarrow \infty$. Thus $h<0$ is true quite generally.

We have shown that in a theory regulated by a general function $\mathcal{K}\left(q^{2} / \Lambda^{2}\right)$, a negative kinetic term for $\Phi$ can be generated by choosing a large enough bare coupling $g_{0}^{2} N$ and a value for $m_{\psi}$ of order $\Lambda$ such that $h(z)<0$. For large $\Lambda$, higher dimension operators are suppressed by $\Lambda^{2}$. As discussed in Sec. III, introducing other scalars coupled to $\Phi$ can give rise to a minimum for $P(X)$ by generating, at the classical level, a correction to $P(X)$ that is monotonically increasing.
[1] S. Weinberg, Phys. Rev. Lett. 59, 2607 (1987). See also H. Martel, P. R. Shapiro, and S. Weinberg, Astrophys. J. 492, 29 (1998).

[2] S. Kachru, R. Kallosh, A. Linde, S. P. Trivedi, Phys. Rev. D 68, 046005 (2003); L. Susskind, hep-th/0302219; M. R. Douglas, J. High Energy Phys. 05 (2003) 046; T. Banks, M. Dine, and E. Gorbatov, J. High Energy Phys. 08 (2004) 058;A. Giryavets, S. Kachru, P. K. Tripathy, and S.P. Trivedi, J. High Energy Phys. 04 (2004) 003; M. R. Douglas, hep-ph/0401004; A. Giryavets, S. Kachru, and P. K. Tripathy, J. High Energy Phys. 08 (2004) 002; see also, T. Banks, hep-th/0412129.

[3] J. D. Barrow and F. J. Tipler, The Anthropic Cosmological Principle (Oxford University Press, Oxford, 1986); M. Rees Complexity 3 (1997);V. Agrawal, S. M. Barr, J. F. Donoghue, and D. Seckel, Phys. Rev. D 57, 5480 (1998); M. Tegmark and M.J. Rees, Astrophys. J. 499, 526 (1998); M. J. Rees, astro-ph/0401424; see also, L. Smolin, hep-th/0407213.

[4] I. Zlatev, L. M. Wang, and P. J. Steinhardt, Phys. Rev. Lett. 82, 896 (1999); P. J. Steinhardt, L. M. Wang, and I. Zlatev, Phys. Rev. D 59, 123504 (1999); I. Zlatev and P.J. Steinhardt, Phys. Lett. B 459, 570 (1999).

[5] P. J.E. Peebles and B. Ratra, Astrophys. J. 325, L17 (1988); B. Ratra and P. J.E. Peebles, Phys. Rev. D 37, 3406 (1988); R. R. Caldwell, R. Dave, and P. J. Steinhardt, Phys. Rev. Lett. 80, 1582 (1998); J. A. Frieman, C. T. Hill, A. Stebbins, and I. Waga, Phys. Rev. Lett. 75, 2077 (1995); P. G. Ferreira and M. Joyce, Phys. Rev. Lett. 79,
4740 (1997); P. G. Ferreira and M. Joyce, Phys. Rev. D 58, 023503 (1998).

[6] R. Fardon, A.E. Nelson, and N. Weiner, J. Cosmol. Astropart. Phys. 10 (2004) 005; D. B. Kaplan, A.E. Nelson, and N. Weiner, Phys. Rev. Lett. 93, 091801 (2004); N. Afshordi, M. Zaldarriaga, and K. Kohri, Phys. Rev. D 72, 065024 (2005); R. Fardon, A.E. Nelson, and N. Weiner, hep-ph/0507235.

[7] S. Weinberg, Rev. Mod. Phys. 61, 1 (1989).

[8] S. R. Coleman, Nucl. Phys. B310, 643 (1988).

[9] J. Polchinski, Phys. Lett. B 219, 251 (1989).

[10] N. Arkani-Hamed, H. C. Cheng, M. A. Luty, and S. Mukohyama, J. High Energy Phys. 05 (2004) 074.

[11] N. Arkani-Hamed, P. Creminelli, S. Mukohyama, and M. Zaldarriaga, J. Cosmol. Astropart. Phys. 04 (2004) 001; S. L. Dubovsky, J. Cosmol. Astropart. Phys. 07 (2004) 009; M. Peloso and L. Sorbo, Phys. Lett. B 593, 25 (2004); B. Holdom, J. High Energy Phys. 07 (2004) 063; N. Arkani-Hamed, H.C. Cheng, M. Luty, and J. Thaler, J. High Energy Phys. 07 (2005) 029; D. Krotov, C. Rebbi, V. A. Rubakov, and V. Zakharov, Phys. Rev. D 71, 045014 (2005); L. Amendola, Phys. Rev. Lett. 93, 181102 (2004); S. Mukohyama, Phys. Rev. D 71, 104019 (2005); M. V. Libanov and V.A. Rubakov, J. High Energy Phys. 08 (2005) 001; N. Arkani-Hamed, H. C. Cheng, M. A. Luty, S. Mukohyama, and T. Wiseman, hep-ph/0507120.

[12] S. Coleman, Aspects of Symmetry (Cambridge University Press, Cambridge, England, 1985).

[13] J. Polchinski, Nucl. Phys. B231, 269 (1984). 